\title{
RT-FOCUSS Algorithm for Sparse Recovery in Fully-perturbed Linear Model
}

\author{
Xuebing $\operatorname{Han}^{1, a^{*}, \text { Zhaojun Jiang }}{ }^{2, b}$ \\ ${ }^{1,2}$ Air Force Airborne Academy, Guilin, P.R.China \\ ahanxuebing-08@tsinghua.org.cn, bijjhi@126.com
}

\begin{abstract}
Keywords: underdetermined system, fully-perturbed linear model, sparse recovery, RT-FOUCSS. Abstract: In this paper, an improved and regularized algorithm is proposed to solve the sparse recovery problem in "fully-perturbed" model, which means perturbations present both in measurements and dictionary matrix. The paper shows how the regularized algorithm is derived based on TLS (total least-squares) and FOCUSS (FOCal Underdetermined System Solver) methods. In the end, simulations illustrate the advantages of the new algorithm.
\end{abstract}

\section{Introduction}

The problem of finding sparse solutions to underdetermined system of linear equations has been a hot spot of researches in recent years, because of its widespread application in compressive sensing/sampling (CS), biomagnetic imagining, source localization, signal reconstruction, etc. In the noise-free setup, CS theory holds promise to explain the equivalence between $\mathrm{I}_{0}$-norm minimization and $\mathrm{I}_{1}$-norm minimization as solving exactly linear equations when the unknown vector is sparse[1]. Variants of CS for "noise setup" of perturbed measurements are usually solved based on convex optimization[2], greedy algorithms[3] or FOCUSS[4].

Recently, only a few attentions had been paid on the sparse problems in fully-perturbed linear model. To solve this problem, performance analysis of CS for the linear regression model under sparsity constraints was researched in ref. [5], S-TLS was devised to reconstruct sparse vectors based on convex optimization[6], SD-FOCUSS evaluated sparse solutions and perturbation matrix synchronously through an iterative and convergent process[7], and TLS-FOCUSS reduced the impact of perturbations both in measurements and dictionary matrix based on TLS and FOCUSS methods to find the sparse solutions[8]. However, the research of ref. [5] is limited in theoretical aspect and does not devise systematic approaches; S-TLS is unsuitable for large scale problems because of highly-computational burden; due to needing to estimate perturbations, which is impossible actually, SD-FOCUSS is just a suboptimal algorithm. Different of above algorithms, TLS-FOCUSS is low-computational, efficient and optimal. However, it is short of practicability with assuming that perturbations containing two different categories are i.i.d. (independently and identically distributed).

The objective of this paper is to extend the TLS-FOCUSS algorithm so that it can be used in the fully-perturbed linear model where measurement-noise and matrix perturbations belong to different types. It is shown how an adjustment to the parameters of system model leads to a regularized version of TLS-FOCUSS algorithm, RT-FOCUSS algorithm.

\section{Perturbed Linear Regression Model}

For the underdetermined linear model $\mathbf{y}=\mathbf{A x}$, where the unknown $n \times 1$ vector $\mathbf{x}$ is to be recovered from the given $m \times 1$ measurements vector $\mathbf{y}$ and the $m \times n$ matrix $\mathbf{E}$, CS theory asserts that it has the unique solutions with $\mathbf{x}$ being sparse and $A$ satisfying some property[1]. Then consider the perturbed linear regression model with perturbations in measurements and dictionary[6-8], which can be formulated as

$$
\mathbf{y}=(\mathbf{A}+\mathbf{E}) \mathbf{x}+\mathbf{e},
$$


where $\mathbf{e} \in \mathbf{R}^{m}$ represents the measurement-noise vector, $\mathbf{E} \in \mathbf{R}^{m \times n}$ represents the perturbations matrix, and usually $m=n$. Due to randomness and uncertainty, it is a reasonable assumption that the components of noise or perturbations in the same channel are independently and identically Gaussian distributed, i.e. $\mathbf{e} \sim N\left(0, \sigma_{1}^{2} \mathbf{I}\right)$ and $\operatorname{vec}(\mathbf{E}) \sim N\left(0, \sigma_{2}^{2} \mathbf{I}\right)$, where vec $(\cdot)$ is matrix vectorizing operator. With assuming that $\mathbf{v} \sim \mathbf{N}\left(\mathbf{0}, \sigma^{2} \mathbf{I}\right)$, i.e. $\sigma_{1}=\sigma_{2}$, the solutions of TLS-FOCUSS algorithm has been reconstructed as $\arg \min _{\mathbf{z}}\|\mathbf{B} \mathbf{z}\|_{2}^{2} /\|\mathbf{z}\|_{2}^{2}+\gamma(\sigma, p)\|\mathbf{z}\|_{p}^{p}$, where $\mathbf{B}=[-\mathbf{y}, \mathbf{A}], \quad \mathbf{z}=\left[\begin{array}{l}1 \\ \mathbf{x}\end{array}\right], 0<p \leq 1$, and $\gamma(\sigma, p)$ is sparsity-tuning parameter depending on $\sigma$ and $p$ [8]. However, how does TLS-FOCUSS algorithm work effectively if $\sigma_{1} \neq \sigma_{2}$ ?

\section{Regularized TLS-FOCUSS (RT-FOCUSS) Algorithm}

In the maximum a posteriori (MAP) sense, the estimate of $\mathbf{x}$ can be obtained as

$$
\mathbf{x}_{\mathrm{MAP}}=\arg \max _{\mathbf{x}} \ln p(\mathbf{x} \mid \mathbf{y})=\arg \max _{\mathbf{x}}[\ln p(\mathbf{y} \mid \mathbf{x})+\ln p(\mathbf{x})] \text {. }
$$

And the elements of sparse $\mathbf{x}$ can be assumed to be distributed as general Gaussian and independent, which is

$$
p(\mathbf{x})=C_{1} \exp \left(-\frac{1}{2 \beta^{p}} \sum_{k=1}^{m}|\mathbf{x}[k]|^{p}\right)
$$

where $C$ represents constant, $0<p \leq 1$ and $\beta=2^{-\frac{p}{2}} \frac{\Gamma(1 / p)}{\Gamma(3 / p)}$ ( $\Gamma(\cdot)$ means Gamma function)[4]. Formula (1) can be rewritten as $\mathbf{y}-\mathbf{A x}=\mathbf{G}(\mathbf{x}) \mathbf{v}$, where $\mathbf{G}(\mathbf{x})=\left[\sigma_{1}, \sigma_{2} \mathbf{x}^{T}\right] \otimes \mathbf{I}_{m \times m}$, $\mathbf{v}=\operatorname{vec}\left(\left[\mathbf{e} / \sigma_{1}, \mathbf{E} / \sigma_{2}\right]\right)$, and $\otimes$ represents Kronecker product.. With $\mathbf{v} \sim \mathbf{N}(0,1)$ and $\mathbf{G}(\mathbf{x}) \mathbf{G}^{H}(\mathbf{x})=\left(\sigma_{1}^{2}+\sigma_{2}^{2}\|\mathbf{x}\|_{2}^{2}\right) \mathbf{I}$, it can be obtained that

$$
\ln p(\mathbf{y} \mid \mathbf{x})=\frac{1}{2} \frac{(\mathbf{y}-\mathbf{A x})^{H}(\mathbf{y}-\mathbf{A x})}{\sigma_{1}^{2}+\sigma_{2}^{2}\|\mathbf{x}\|_{2}^{2}}+C_{2}
$$

From (2), (3) and (4) one can proceed to find the MAP estimate as

$$
\mathbf{X}_{\mathrm{MAP}}=\arg \min _{\mathbf{x}}\left[\frac{\|\mathbf{y}-\mathbf{A} \mathbf{x}\|_{2}^{2}}{\sigma_{1}^{2}+\sigma_{2}^{2}\|\mathbf{x}\|_{2}^{2}}+\frac{\|\mathbf{x}\|_{p}^{p}}{\beta^{p}}\right]
$$

Let $\mathbf{B}=\left[-\mathbf{y} / \sigma_{1}, \mathrm{~A} / \sigma_{2}\right], \mathbf{z}=\left[\begin{array}{l}\sigma_{1} \\ \sigma_{2} \mathbf{x}\end{array}\right]$, and formula (5) can be rewritten as

$$
\hat{\mathbf{z}}_{M A P}=\arg \min _{\mathbf{z}}\|\mathbf{B} \mathbf{z}\|_{2}^{2} /\|\mathbf{z}\|_{2}^{2}+\gamma\|\mathbf{z}\|_{p}^{p},
$$

where $\gamma=\frac{1}{\beta^{p} \sigma_{2}^{p}}$. Then formula (6) is identified as the form of TLS-FOCUSS algorithm. Through simplification, the objective function of (6) is equivalent to

$$
\min _{\mathbf{z}}\left[\|\mathbf{B} \mathbf{z}\|_{2}^{2}+\gamma\|\mathbf{z}\|_{p}^{p}\right], \quad \text { s.t. }\|\mathbf{z}\|_{2}^{2}=1
$$

Using Lagrange multiplier method, the objective function can be rewritten as

$$
J(\mathbf{z})=\|\mathbf{B} \mathbf{z}\|_{2}^{2}+\gamma\|\mathbf{z}\|_{p}^{p}+\lambda\left(1-\mathbf{z}^{H} \mathbf{z}\right)
$$

where $\lambda$ is the Lagrange multiplier. The optimal solution $\mathbf{z}_{*}$ must satisfy $\nabla_{\mathbf{z}} T\left(\mathbf{z}_{*}\right)=0$, so one can get $\left(\mathbf{B}^{H} \mathbf{B}+\alpha \Pi^{-1}\left(\mathbf{z}_{*}\right)\right)^{-1} \mathbf{z}_{*}=\frac{1}{\lambda} \mathbf{z}_{*}$, where $\alpha=p \gamma / 2, \Pi(\mathbf{z})=\operatorname{diag}\left(\left[|\mathbf{z}[i]|^{2-p}\right]_{i=1, \mathrm{~L}, n+1}\right)$. This suggests the 


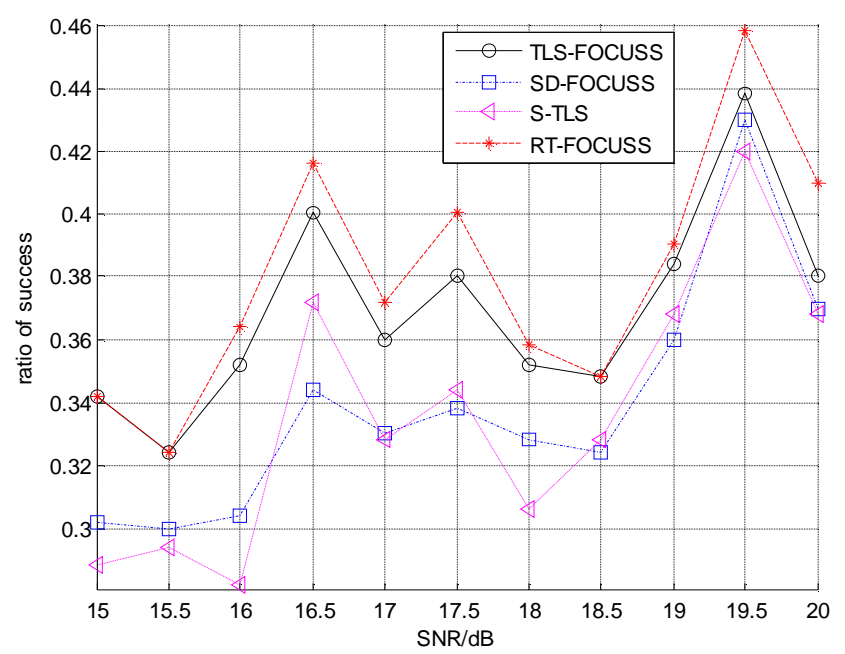

Fig. 1 success probability of algorithms in finding the nonzero indices

following iterative relaxation algorithm:

$$
\Phi\left(\mathbf{z}_{k-1}\right) \mathbf{z}_{k}=\frac{1}{\lambda} \mathbf{z}_{k}
$$

where $\Phi\left(\mathbf{z}_{k-1}\right)=\left(\mathbf{B}^{H} \mathbf{B}+\alpha \Pi^{-1}\left(\mathbf{z}_{k-1}\right)\right)^{-1}$. Thus $1 / \lambda$ should be the maximal eigenvalue of $\Phi\left(\mathbf{z}_{k-1}\right)$ to guarantee $\lambda$ being the minimal value, and the corresponding eigenvector is chosen as the $k$-th estimate of $\mathbf{z}$. To reduce the dimensions of computation, matrix inversion formula can be used to get $\Phi(\mathbf{z})$ in each iteration procedure, which is

$$
\Phi=\frac{1}{\alpha}\left(\Pi-\Pi \mathbf{B}^{H}\left(\alpha I-\mathbf{B} \Pi \mathbf{B}^{H}\right)^{-1} \mathbf{B} \Pi\right) .
$$

When the convergent solution $\mathbf{z}_{*}$ is obtained, one can get $\mathbf{x}_{*}=\frac{\sigma_{1} \cdot\left[z_{*_{2}}, \mathrm{~L}, z_{*_{n+1}}\right]^{T}}{\sigma_{2} \cdot z_{*_{1}}}$.

\section{Simulation Results}

In this section, computer simulations are conducted to evaluate the performance of RT-FOCUSS algorithm and other similar algorithms used for fully-perturbed model. The $m \times n$ matrix $A$ is created with entries being each Gaussian random variables with mean 0 and variance 1 . To evaluate the methods, the true sparse solution has to be known, which is often hard to know actually. Then a known sparse vector $\mathbf{x}$ is created with only $s$ rows being nonzero entries. The indices of the $s$ nonzero rows are chosen randomly from a discrete uniform distribution, and the amplitudes of these indices are chosen randomly from a standard normal distribution. Under this situation, the signal-to-noise ratio (SNR) is defined as $S N R=-10 \log \sigma_{1}^{2}$, and dictionary-to-perturbation ratio (DPR) is defined as $D P R=-10 \log \sigma_{2}^{2}$. The parameters of simulation are set as follows: $p=0.5, m=20, n=128, s=3$. In each Monte Carlo experiment, 500 trials are carried independently. In the following simulations, besides RT-FOCUSS algorithm, other similar algorithms are involved: S-TLS[6], SD-FOCUSS[7], TLS-FOCUSS[8]. The range of SNR is set from $15 \mathrm{~dB}$ to $20 \mathrm{~dB}$, and DPR is fixed to $15 \mathrm{~dB}$. In a single simulation, the algorithm is considered to be successful if all nonzero indices of $\mathbf{x}$ are found exactly; otherwise, it is considered to be failed.

Fig. 1 shows the statistical results of success percentage. It can be seen that RT-FOCUSS algorithm presents more robust performance than other algorithms because of its higher success probability. Fig. 2 shows the statistical RMSE (root-mean-square error) curves of signal-amplitude recovery when algorithms find nonzero indices of $\mathbf{x}$ correctly. One can find that the recovery result of RT-FOCUSS algorithm is much closer to the real inputs than the results of other algorithms. 


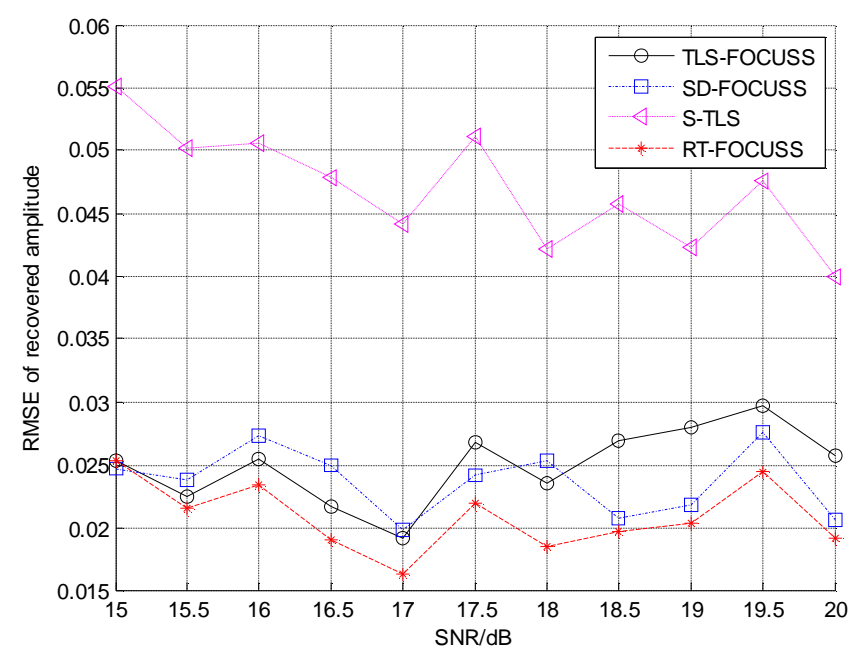

Fig. 2 RMSE of signal amplitude recovery

\section{Conclusions}

In this paper, the problem of sparse recovery in fully-perturbed environments has been tackled. Against different types of perturbations exiting in the measurements and dictionary, a new algorithm, RT-FOCUSS, has been proposed to solve this problem. We described how to derive RT-FOCUSS algorithm and the principle to restrain the different perturbations. Simulations proved that RT-FOCUSS algorithm can better identify the generating sparse data than previous algorithms. The benefits of RT-FOCUSS algorithm make it a good choice of sparse recovery for practical applications.

\section{Acknowledgements}

This work was supported in part by the Guangxi Natural Science Foundation of China (No. 2014GXNSFBA118285).

\section{References}

[1] E. Candes, T. Tao, Decoding by linear programming, IEEE Trans. Inf. Theory 51 (2005) 4203-4215.

[2] E. Candes, M. Wakin and S. Boyd. Enhancing Sparsity by Reweighted I Minimization. J Fourier Anal Appl. Vol. 14 (2008), p. 877-905.

[3] D. Needel, R. Vershynin. Signal Recovery From Incomplete and Inaccurate Measurements Via Regularized Orthogonal Matching Pursuit. IEEE J. Selected Topics Signal Process. Vol. 4 (2010), p. 310-316.

[4] B. D. Rao, K. Engan, S. F. Cotter, J. Palmer, and K. Kreutz-Delgado. Subset selection in noise based on diversity measure minimization. IEEE Trans. Signal Process.. Vol. 51 (2003), p. 760-770.

[5] M. Herman and T. Strohmer. General Deviants: An Analysis of Perturbations in Compressed Sensing. IEEE Journal of Selected Topics in Signal Process.. Vol. 4 (2010), 342-349.

[6] H. Zhu, G. Leus, and G. B. Giannakis. Sparsity-cognizant total least-squares for perturbed compressive sampling. IEEE Trans. Signal Process.. Vol. 59 (2011), p. 2002-2016.

[7] X. Han and H. Zhang. Research of sparse recovery algorithm based on model noise. Journal of Electronics \& Information Technology. Vol. 34 (2012), p.1813-1818.

[8] X. Han H. Zhang and H. Meng. TLS-FOCUSS for sparse recovery with perturbed dictionary. In Proceedings of the International Conference on Acoustics, Speech, and Signal Processing. (2011), 3952-3955. 